\title{
LÓGICA MODAL MEGÁRICO-ESTOICA
}

\section{Posibilidad y necesidad como operadores aléticos}

Megaric-Stoic logic: possibility and necessity as alethic operators

\author{
José AlejandRo Fernández Cuesta
}

Universidad Rey Juan Carlos y Universidad de Salamanca

\section{KEY WORDS}

Megaric-Stoic logic Accessibility relationship True logical constants History of modal logic Ancient Greek logic Philosophy of modal logic

\section{ABSTRACT}

In this article we introduce a possible way to interpret the possibility and necessity notions developed within the Megaric-Stoic logic as alethic modal operators. Megaric-Stoic semantics will be introduced as metaphysical background for necessity and possibility definitions and arguments will be offered in order to give up the prevailing interpretations which include ad hoc variables. After proposing the survey of diodoric definitions from relational modal semantics, a series of topics that deserve being revisited from this perspective will be pointed out.
PALABRAS CLAVE

Lógica megárico-estoica Relación de accesibilidad Verdaderas constantes lógicas Historia de la lógica modal Lógica de la Antigua Grecia Filosofía de la lógica modal

\section{RESUMEN}

En este artículo presentamos una posible vía para interpretar las nociones de posibilidad y necesidad desarrolladas en el seno de la lógica megárico-estoica como operadores modales aléticos. Se introducirá la semántica megárico-estoica como trasfondo metafísico de las definiciones de necesidad y posibilidad y se ofrecerán argumentos para abandonar las interpretaciones predominantes que incluyen variables temporales ad hoc. Tras proponer la lectura de las definiciones diodóricas desde una semántica modal relacional se señalará una serie de temas que merecen ser revisitados desde esta óptica. 


\section{Introducción}

$\mathrm{E}$ l estudio de la historia de la lógica modal suele enfocarse, aunque no de manera explícita, desde dos ángulos fácilmente identificables que, en último término, responden a dos posturas interpretativas concretas respecto de lo que ha de considerarse lógica modal propiamente hablando y aquello que no.

Por un lado, encontramos lo que podríamos llamar historia de la lógica modal contemporánea. Esta se centraría en el estudio de los cálculos modales desarrollados a partir de la famosa publicación de Lewis (1918) y abarcaría tanto los desarrollos de las lógicas con operadores aléticos como, en general, otro tipo de lógicas susceptibles de ser consideradas modales -epistémicas, temporales, condicionales o multivalentes entre otras. Aquí, por lógica modal se entiende, principalmente, el conjunto de los avances técnicos desarrollados durante los últimos años; especialmente en lo relativo a la demostración de teoremas de completitud y corrección primero y en lo relativo a su extensión a cálculos algebraicos en segundo lugar. Aunque la tendencia, desde los años 70, ha sido la de incorporar un número creciente de discusiones metafísicas en este contexto, el núcleo del objeto de la lógica modal no dejará de estar formado principalmente por los propios cálculos formales.

Por otro lado, encontramos el ámbito al que tendría sentido llamar historia clásica de la lógica modal. Pues, si bien es cierto que el desarrollo de los cálculos modales contemporáneos arranca con Lewis (1918), también es cierto que los operadores de necesidad y posibilidad se han estudiado como categorías metafísicas encuadradas en contextos lógicos -entendiendo lógica en un sentido histórico-filosófico amplioya desde la Antigua Grecia. Así, en orden cronológico inverso, encontramos que Frege, aunque descarte para su conceptografía la incorporación de nociones modales, sí que las tiene presentes en cuanto que categorías conceptuales $\mathrm{y}$, por tanto, eminentemente lógicas. Esta tradición vio su mayor exponente en la deducción trascendental de las categorías kantianas. En ella, Kant sintetiza los estándares lógicos desarrollados desde la Edad Media dentro del marco aristotélico-tomista al incorporar los juicios de modalidad problemáticos, apodícticos y asertóricos. Pero el verdadero origen de la lógica modal en cuanto que disciplina encargada de aproximarse a los conceptos de necesidad y posibilidad desde una metodología lógica o dialéctica la encontramos, sin duda, en la escuela de Megara.

Debemos puntualizar, además, que ambos recorridos históricos de la lógica modal -el iniciado por los megáricos por un lado y el iniciado por Lewis por otro- aunque mantengan obviamente una relación de sucesión cronológica, no se encuentran ligadas por una relación de sucesión lógica.

Todo apunta a que los precursores de los cálculos modales contemporáneos fueron mayoritariamente ajenos a los desarrollos megáricos $\mathrm{y}$ estoicos $\mathrm{y}$, sin embargo, encontramos al menos dos paralelismos lo suficientemente relevantes como para ser tenidos en cuenta: (i) que en ambos momentos históricos primero se configuran y desarrollan metodologías formales -generalizables- a partir de análisis lógicos del lenguaje natural para, a continuación, desde los resultados obtenidos a la luz de dicha metodología, iniciar un estudio de las consecuencias metafísicas más relevantes y (ii) que en ambos casos se apremia la caracterización de los operadores modales de necesidad y posibilidad como constantes lógicas interdefinibles a partir de una función -implícita en el caso megárico-estoico por cuanto su semántica aún se centra en la distinción sujetoobjeto- de accesibilidad -explícita en el contexto contemporáneo una vez ha cuajado el proceder fregeano de acercarse a las proposiciones desde la clave función-argumento y se han dotado los cálculos de semánticas relacionales especialmente a partir de los trabajos de Kripke (1963) y Hintikka (1962).

En el presente artículo propongo una fundamentación genealógica de la historia clásica de la lógica modal que, sin embargo, tenga presente también los resultados obtenidos durante el último siglo a partir de los avances técnicos de las semánticas modales. Para ello, mencionaré de pasada cómo poco a poco se fragua, desde la conocida como tradición eleática de la filosofía presocrática, la incorporación de 
las nociones de necesidad y posibilidad como conceptos ya eminentemente filosóficos en una tecnificación susceptible de ser catalogada como lógica -en el sentido más aceptado del término. Resumiré el contexto semántico en que las escuelas de Megara y la Estoa llevan a cabo sus respectivos análisis de estas nociones y expondré sus definiciones técnicas de ambos conceptos a partir de las posturas de Diodoro y Filón. Esto último lo haré teniendo en cuenta las formalizaciones propuestas por Mates (1973) y Bocheński (1963) actualizadas por Benítez (2015) y, finalmente, propondré una nueva vía de formalización de ambas nociones desde una semántica relacional que permita entrever en los desarrollos megárico-estoicos, al igual que en los contemporáneos, cómo la relación o función de accesibilidad ha de ser tenida en cuenta como una verdadera constante lógica.

\subsection{Pre-historia modal: la tradición eleática}

Ya en Ferécides (Damascio, De principiis 124 bis) son rastreables algunas de las primeras incorporaciones en contextos abstractos reflexivos de términos modales como la posibilidad o la necesidad. No obstante, su uso aún no se había cargado de una significación filosófica lo suficientemente profunda y tecnificada como para poder hablar aquí de una prehistoria de la lógica modal. Habría que esperar hasta Anaximandro para encontrar, en su famoso pasaje conservado por Teofrasto en su Opiniones de los físicos y por Simplicio, Fis. 24, 17, una alusión explícita a la noción de necesidad desde un sentido contractual, entendida como obligación- cuando se afirmó, en referencia a lo indeterminado (tó ápeiron), que es en ello donde acaece la destrucción de las cosas por necesidad (katà tò khreón).

En el mismo sentido, Heráclito opuso a la discordia (éris), como elemento motriz, la necesidad (khréon). Pero aquí la significación de lo que convenimos en traducir por necesidad, aun no es tal, y simplemente se está refiriendo a un tipo concreto de necesidad, bien física o bien cosmológica, que ha de ser tomada antes como fuerza mayor que como una marca lógica que indique aquello que permanezca invariante.
Es en Parménides $\mathrm{y}$, posteriormente en Empédocles, donde nos encontramos con un gran salto. En el famoso Poema observamos uno de los testimonios más antiguos en los que se distingue, por primera vez, entre una necesidad en el sentido de fuerza mayor y la necesidad como Ananké, es decir, como divinidad que, en último término, es responsable de la cohesión cosmológica y que rige el orden de la naturaleza acorde a la justicia.

Esta última noción, como abstracción deificada de la necesidad será la que finalmente permee en la historia de la filosofía hasta, en su vertiente de análisis ontológico, dar lugar a lo que hoy trabajamos como necesidad en cuanto que operador modal. La necesidad como invarianza es la generalización -forma-lizaciónde la uis maior reconocible en cualquier lenguaje natural y en el contexto de análisis filosófico esta generalización ha pasado indefectiblemente por su deificación -aun cuando la deidad pueda ser anterior a su incorporación en una cosmología presocrática, que dicha tecnificación se dé en una cosmología y no una cosmografía, es lo que permitirá que la aproximación a su caracterización más formal parta de su conceptualización primigenia.

Ananké determina, ya desde Homero, que algo es, como sea, por fuerza. Y son rastreables hasta cuatro vertientes semánticas en el uso de este término: una física, una moral, una lógica y una cosmológica. La última, en íntima relación con la necesidad física, se inserta en la tradición órfica hasta cobrar un mayor peso en Filolao y la corriente pitagórica; la presencia del término en el Papiro de Derveni nos permite entender que se fraguó como noción filosófica semánticamente tecnificada en el Timeo de Platón, aunque su inclusión en la reflexión cosmológica abstracta se deba al atomismo de Leucipo y Demócrito.

Pero la caracterización de este uso del término necesidad, aún distante de nuestra noción lógica contemporánea, comenzaría a aproximarse al operador modal actual desde el trato que de ella configuró Zenón de Elea, quien, en último término, impregnó un giro técnico en la utilización de la noción en un contexto filosófico eminentemente lógico -DK 291, B1 2-4, 10 o 293 5, entre otros. 
Por tanto, el origen de la lógica modal, en cuanto que tal, para una genealogía clásica que focalice el interés del estudio de la noción de necesidad -y consecuentemente de la posibilidad-dentro de un contexto que primase el análisis conceptual desde una postura principalmente inferencial, lo tenemos que rastrear en la tradición eleática. Primero en Parménides, pero, de manera más notoria, en Zenón y, posteriormente, en Gorgias -DK 82 B 3, 38 y 70. Todo esto, sin olvidar que la escuela de Megara era llamada también dialéctica por su adscripción al programa eleático -antes que al jonio o al pitagórico, como pudiera ser el caso, en las escuelas socráticas, de Fedón o Platón respectivamente.

\subsection{La vertiente aristotélica}

Por otro lado, si bien Aristóteles se hizo cargo de las nociones modales dentro de un contexto silogístico más amplio, susceptible de tomarse como lógico, debemos realizar una serie de matizaciones respecto de los desarrollos aristotélicos de la modalidad, aunque el más importante de todos ellos será que, finalmente, la base metafísica operante de fondo tras su propuesta, mucho más restringida que la megárica, generó una serie de problemas de difícil resolución.

Aristóteles no prestó especial atención a las problemáticas modales dentro de la modulación categórica de los silogismos, aunque sí que dedicó unas cuantas reflexiones a su análisis dentro de un contexto algo más amplio. Las principales fuentes son el Sobre la Interpretación, XII y XIII, los Analíticos Primeros I, 3 y 13 y I, 822. Aquí, la modalidad pertenece a la teoría de la argumentación y su caracterización como noción, más pragmática que conceptual, engarza directamente con el rechazo fregeano a las semánticas modales -que hoy ve su mayor apoyo en las posturas que toman los operadores modales como operadores de cambio de circunstancia.

En primer lugar, para Aristóteles, la contradictoria de «es necesario que $p$ » usaremos $L$ y $M$ para representar respectivamente los operadores de necesidad y posibilidad- no sería $M \neg p$ sino $\neg M p$. Esto se debe a que las modalidades afectan al contenido de toda oración y no solamente a una parte proposicional de esta. De hecho, en Sobre la Interpretación XIII 22 b 20 Aristóteles explicita la interdefinición de los operadores modales que hoy día usamos: $M p=\neg L \neg p$.

$\neg M p$ equivale a $L \neg p$, mientras que $M \neg p$ a $\neg L p \mathrm{y}$, por tanto, tiene sentido que la contradictoria sea la que de manera explícita implica la asunción de la negación de la oración negada. Si, hasta aquí, la propuesta aristotélica es prácticamente análoga a la resultante de la interdefinición de operadores modales actual -es del todo compatible como, de hecho, lo será también la fundamentación de la silogística con los desarrollos megáricos-, entonces el problema surge una vez se ha restringido el contenido semántico de dichos operadores: la inserción de las modalidades dentro de la propuesta esencialista hilemórfica fuerza que se genere una grave confusión entre posibilidad y contingencia. Por mor de la claridad explicativa, aun cuando estemos cayendo en una simplificación anacrónica, entenderemos posibilidad, en sentido amplio, como la aserción de un contenido juzgable en algún mundo posible entendido en sentido amplio. Siguiendo la línea semántica propuesta por Kripke (1963), tomaremos la noción de mundo posible como mera estipulación contrafáctica o construcción subjuntiva. Por el contrario, interpretaremos contingencia en su sentido restricto más extendido tanto en la Edad Media como en la Modernidad -especialmente en la polémica Leibniz-Kant- como posibilidad espacio-temporal. Es decir, contingente será, grosso modo y desde una perspectiva modal, nada más y nada menos que aquello que no es necesario físicamente que no sea.

Lo anterior supone el trasfondo de la aparente contradicción en que incurre Aristóteles y que los Kneale (1962) señalan. Aunque el estagirita parta, en Sobre la Interpretación XIII 22 a, 15, de que no es lo mismo posible (dýnaton) que contingente (endekhómenon), asumirá, sin embargo, como ya se ha podido entrever en base al uso de los términos, la modalidad como contingencia. Para Aristóteles:

1) Si $p$ es contingente, entonces no- $p$ también lo es.

2) Si $p$ es necesario, entonces no es necesario no- $p$. Es decir, es contingente que $p$. 
3) Por tanto, si es necesario que p, entonces es contingente no- $p \mathrm{y}$, por tanto, posible. Lo cual entabla una contradicción.

La contradicción radica, claramente, en la equivalencia entre contingencia y posibilidad. De hecho, restringir la contingencia a un tipo de posibilidad termina forzando la asunción que, más allá de su caracterización en un marco eminentemente espacio-temporal, se siga una lectura de esta como mera indeterminación posibilitista. Para Aristóteles, la contingencia prima a la posibilidad y eso permite que se infiera una contradicción como la anterior.

Contingente, en el sentido modal aristotélico, es todo aquello que no sea necesario, aquello de lo cual, supuesta su existencia, no se fuerce que se siga nada imposible. Y decir que sea posible que no $A$ sea $B$ equivaldrá a afirmar que no es necesario que todo $B$ sea $A$. Lo que debe destacarse de lo anterior es que el análisis de las modalidades en términos de clases, más allá incluso de si se adopta una postura hilemórfica o no, es igualmente problemático. De hecho, si fueron los desarrollos megárico-estoicos los que propugnaron una semántica adecuada para poder definir -e interdefinir- los operadores modales en cuanto que tal, esto se debió a que su semántica propugnó un análisis sobre una lógica de enunciados.

Pues bien, primada la contingencia metafísica a la posibilidad y asumidas las reglas de interdefinición usuales, la contingencia se definirá como la conjunción de dos subcontrarios en el cuadro ontológico aristotélico. Necesario es aquello que se sigue de la necesidad (tò ex anánkes hypárkhein). Y la necesidad se dividirá en ontológica y lógica, aunque finalmente el correlato esencia (tó tí én eínai) - entidad (ousía) no se hará cargo de la diferenciación modal. También distinguirá Aristóteles entre una necesidad simple (aplós) y una óntica (toúton ónton) que han convenido en traducirse como absoluta e hipotética la mayoría de las veces, pero que tampoco quedan lo suficientemente aclaradas.

Posible será aquello que se siga de lo contingente (tò endékhesthai hýparkhein). La posibilidad se dividiría entre una bilateral $o$ contingente hipotética (aóriston) y generalizada o indeterminada (hòs epì tò polý). La asertividad se derivará de la indefinición o simpleza (tò aplós hypárkhein) de manera no menos confusa.

Lo que sin duda debemos apuntar también, antes de pasar a analizar la postura megárica, es que cuando Aristóteles, por ejemplo, critica a estos en su Metafísica lo hace desde sus propias coordenadas y definiciones de necesidad $y$ posibilidad. La definición del movimiento como el paso de la potencia (dýnamis) al acto (enérgeia) es algo inconcebible si se asume la posibilidad como una estructuración meramente formal respecto de lo que hemos llamado estipulaciones contrafácticas. Y es curioso hacer notar, aunque su desarrollo habrá de dejarse para otro momento, cómo la réplica megárica será análoga a nuestra crítica al paso inferencial de los argumentos ontológicos, especialmente a los formulados en la línea de San Anselmo en su Proslogion.

\section{Fundamentos de la lógica megárico- estoica}

\subsection{Contexto semántico}

Tal y como explica Mates (1973 pp. 27-28), «según los estoicos, hay tres cosas en conexión: 1) el significante o signo, 2) el significado y 3) lo que existe». Para el primero el término más habitual era el de sonido (phoné), lo cual hace alusión a su clara vinculación lingüística. No es de extrañar que la representación simbólica fuera con el sonido antes que con una grafía puesto que la escritura en aquel contexto era algo relativamente reciente en lo que a la discusión filosófica respectaba y muchos filósofos continuaban siendo ágrafos -tal es el archiconocido caso de Sócrates, que no debemos olvidar fue maestro de Euclides, fundador de la escuela megárica.

Aquello que existe remitiría, en segundo lugar, a los objetos que, en un sentido restringido de existente, parece que se identificarían principalmente con las cosas (tá prágmata) en el sentido de entidades locativas.

El significado, sin embargo, entraña una de las mayores complejidades técnico-interpretativas de la filosofía estoica en general. Los estoicos diferenciaban lékta conmemorativos e indicativos por un lado y completos y deficientes 
por otro. Los conmemorativos refieren a una relación observable con el objeto mientras que los indicativos no nombrarían algo perceptible experiencialmente $\mathrm{u}$ observable.

Los significados -proposicionales, podríamos añadir- deficientes serían aquellos divididos en sujeto (ptóseis) y predicado (kategorémata) y se consideraban incompletos. Y es relevante que, tal como Mates (1973 p. 38) apunta, un predicado sería «un lektón deficiente que se combina con un sujeto (en caso nominativo) para formar una proposición».

La doctrina estoica de la verdad, tal y como apunta Bocheński (1963 p. 85), es lo suficientemente confusa, dado el fragmentario estado de las obras que conservamos, como para poder afirmar la práctica imposibilidad de su correcta comprensión. Sabemos que tomaron la verdad como algo concerniente a las proposiciones de manera que si no estaba en ellas sí que debía versar sobre estas. En segundo lugar, una función proposicional se diría verdadera para algunos de sus valores o para todos ellos. En tercer lugar, las representaciones (phantasíai) serían tomadas como verdaderas. Finalmente, un lógos, ya como argumento ya como enunciado, sería tomado como verdadero si, y sólo si, existe una inferencia válida bajo premisas verdaderas. Es decir, la verdad estoica encaja sobre manera con ciertas vertientes inferencialistas del expresivismo lógico contemporáneo. Además, aunque no entraremos en ello puesto que esta presentación de la semántica estoica solamente busca ser una pincelada a modo de contextualización de la caracterización de los operadores modales, se deben subrayar dos aspectos esenciales de la dialéctica megárico-estoica: (i) sus desarrollos constituyen, como ya hemos mencionado, una lógica de enunciados y no de clases -como sucedería en la silogística aristotélica- y (ii) en base a lo anterior se ofrecieron caracterizaciones de las constantes muy cercanas a una definición veritativo-funcional de estas -interdefinición desde conjuntos adecuados incluida.

\subsection{Diodoro y Filón: enfoque tradicional desde la inclusión de variables temporales}

Diodoro, apodado Crono, fue uno de los máximos representantes de las discusiones lógicas en los contextos megáricos. No me centraré en sus definiciones del condicional y las consiguientes discusiones con Filón de Megara -y las ulteriores críticas a ambos de Crisipo y Boecio-, sino que simplemente me limitaré a su definición de los operadores modales. En concreto, era habitual entonces incluir, aun cuando se incorporasen bases semánticas de interdefinición de operadores modales, también los contrarios y los contradictorios. De esta forma, los operadores modales definibles eran cuatro: necesario, posible, imposible e innecesario. Puesto que aquello que es no-posible y aquello que es nonecesario simplemente se forma a partir de la negación de la definición respectiva de posible y necesario, por mor de la simplicidad y claridad expositiva tomaré, como por lo demás es habitual, solamente los operadores L y M y sus definiciones.

Diodoro consideró que una proposición es posible si, y sólo si, es o será -y a continuación se suele sobreentender verdadera- (quod aut est aut erit). Necesario, por otro lado, es aquello que, siendo verdadero, no será falso (quod cum verum sit, non esit falsum). Y Mates (1973) propuso, por tanto, en esta línea, entender las proposiciones modales a través de la inclusión de variables temporales.

Siguiendo los ejemplos propuestos por Alejandro de Afrodisias es posible que esté en Creta si o bien es el caso que ahora mismo lo estoy o bien lo estaré. van Harven (2016), Bocheński (1963) o los Kneale (1962) pero también Prior (1955) y Mates (1973), han seguido esta interpretación. Para ellos, la clave a la hora de abordar estas definiciones de posibilidad y necesidad está en la inclusión de variables temporales en los enunciados.

Cuando Diodoro usa siempre (aei) o a veces (póte), la doctrina contemporánea mayoritaria los interpreta como estrictamente temporales. Y, aunque es también cierto que la partícula póte corresponde antes a un «cuando» que a un «a veces» y la aè̀ a una generalidad que también estaría caracterizada por una cierta indeterminación -suele acompañarse de otras partículas temporales determinativas, siguiendo a Henry George Liddell; Robert Scott (1940) -, también es cierto que los ejemplos expuestos por Diodoro parecen revestir siempre un matiz temporal. 


\section{Modalidades megárico-estoicas como modalidades aléticas}

Sin embargo, tal vez sea pertinente proponer una alternativa en base a las dos principales deficiencias de la aproximación anterior, que por lo demás, conforma la metodología más extendida a la hora de interpretar las nociones modales en base a la inclusión de variables temporales:

1) No es una aproximación semánticamente consistente.

2) No parece que un griego, megárico o estoico, estuviera dispuesto a asumir que dos categorías modales como la necesidad y la posibilidad se restringieran simplemente a un análisis espacio-temporal -tal y como ya hemos mencionado que apuntó Aristóteles.

La formalización de Mates (1973) de la definición de posibilidad sería la que sigue:

$M p=p$ en $t^{\prime} \mathrm{V} E t\left(\mathrm{t}^{\prime}<\mathrm{t} \& p\right.$ en $\left.t\right)$

Es decir, dado un instante $t^{\prime}$ temporal como presente, o bien $p$ es [verdadera] ahora o bien [lo] será en al menos un $t$ posterior. Siguiendo el ejemplo de Alejandro, será posible que esté en Corinto si lo estoy ahora o bien lo estaré en un futuro.

La formulación de Bocheński (1963) original es la siguiente: $p(t n) \vee(\exists t) \cdot t n<t$. $p(t)$. Que, en esencia es la misma que Mates solo que con la notación más extendida en Gran Bretaña en aquel momento.

Por otro lado, la definición de necesidad sería esta:

$L p=p$ en $t^{\prime} \&(t)\left(\mathrm{t}^{\prime}<\mathrm{t} \supset p\right.$ en $\left.t\right)$

Digo que esta aproximación no es semánticamente consistente por un motivo esencial: la ausencia de determinación de una semántica relacional. En un marco clásico de enunciados, tal y como Mates (1973) o Bocheński (1963) lo presentan, no es legítimo, por otro lado, proceder como ellos a partir de la inclusión de variables temporales ad hoc sin caracterización de ningún tipo. Pero, además de dichas variables, existe una relación de orden, aparentemente estricto, que tampoco se puede tomar como constante lógica.

Lo que aquí quiero apuntar es que, entre otras muchas cuestiones, una de las necesidades más acuciantes a la hora de abordar los desarrollos megárico-estoicos modales es la que exige incorporar los formalismos con que los últimos avances en la historia reciente de la lógica modal nos han dotado. Aproximarse a la historia clásica de la lógica modal ajenos a la historia contemporánea de esta, sería un modo de proceder que acabaría suprimiendo de raíz cualquier posibilidad interpretativa de los desarrollos modales clásicos megárico-estoicos como proto-relacionales.

$\mathrm{Y}$ es que hemos visto ya que siempre y a veces en el griego tienen un matiz indeterminado que permiten leerse en un sentido mayor o más amplio que el estrictamente o meramente temporal. Si la relación que existe entre los diferentes estados no es temporal, no solamente eliminamos todos los problemas que generan las formalizaciones propuestas hasta ahora sino, además, una gran parte de las malinterpretaciones de la semántica megáricoestoica misma. En este sentido, no se exige la introducción de ningún orden en un sentido temporal -matiz tal vez introducido desde los comentaristas helenístico, pero sutilmente difuminado de nuevo durante las discusiones medievales en torno a las nociones modales.

Y si la relación entre estos estados de aseverabilidad no es física -espacio-temporal¿Cómo debemos entenderla? La respuesta es que debe comprenderse exactamente tal y como hoy lo hacemos en los diferentes cálculos modales: en su vertiente metafísica. Ya hemos expuesto la clara composicionalidad ontológica de la semántica estoica -más a raíz de la caracterización significativa de las percepciones. Es, sin duda, a todas luces coherente leer las definiciones de operadores modales de Diodoro que hemos expuesto en una semántica relacional y además, hacerlo, sin que el anacronismo hermenéutico sea total. Y esto entronca con el segundo punto que mencionábamos anteriormente como claramente problemático; el relativo a la difícil presuposición de que un megárico $o$ un estoico considerase las definiciones de la posibilidad y la necesidad bajo una restricción puramente temporal, antes que como una extensión metafísico-semántica, algo que pasa por entender las modalidades como operadores modificadores del valor de verdad de 
la oración y no como meros operadores temporales.

En otras palabras, si queremos incorporar los resultados que hemos observado durante el último siglo en el estudio de la lógica y la metafísica modales, debemos, sin duda alguna, abordar las definiciones de necesidad y posibilidad megárico-estoicas como operadores aléticos dotados de una enorme carga metafísica coherente en un contexto semántico concreto y rechazar su lectura desde una formalización inconsistente en un cálculo de enunciados que incluya relaciones de orden total y variables temporales ad hoc.

\section{El argumento soberano de Diodoro: fundamentación metafísica}

Diodoro, personaje clave en la discusión en torno al significado filosófico y esclarecimiento teórico de las nociones modales, desarrolló un argumento, conocido como el argumento soberano para justificar, en último término, la interdefinición los operadores desde un marco eminentemente metafísico. El argumento, con forma de trilema, afirmaba que estos tres enunciados siguientes no pueden ser simultáneamente verdaderos: 1) Toda proposición verdadera sobre el pasado es necesaria. 2) Una proposición imposible no puede seguirse de una proposición posible. 3) Hay una proposición que es posible, pero que no es verdadera ni será verdadera.

Según Epicteto, Diodoro consideró que la tercera proposición era la menos plausible y que, por ello es la que debía ser descartada. En base a este descarte es como se obtendría la definición de lo posible como aquellos que es o será verdadero. Es decir, en último término, la justificación metafísica para la definición de posibilidad en estos términos -análoga a la que hoy manejamos como veremos en la siguiente sección- pasa por asumir que toda proposición posible, aun cuando no afirmada, ha de ser afirmable.

Pero no todos los lógicos antiguos reaccionaron de la misma manera y hubo quienes prefirieron descartar la primera proposición ¿Qué implicaciones tiene esto? No parece descabellado, a priori, abordar las distintas tomas de posición respecto del rechazo de la tercera o la primera afirmación del trilema desde un marco semántico relacional que explicite cómo se relacionan estas posturas con el debate en torno a la definición, con mayores o menores restricciones, de una relación de accesibilidad -y consecuente configuración de cada uno de los distintos cálculos modales normales. Es más, cuando uno atiende a la importancia que el inferencialismo jugó dentro del estoicismo, cuestión que no hemos podido desarrollar en profundidad, es difícil ignorar la urgencia de reestructurar la semántica modal -y en general la metafísica- megárico-estoica desde un contexto metafísico modal contemporáneo.

El rechazo de la primera proposición dependerá del cálculo por el que optemos. En otras palabras, dependiendo de cómo restrinjamos la relación de accesibilidad entre mundos posibles estaremos en disposición de afirmar o negar la primera de las proposiciones del trilema. Puesto que el debate en torno a la interrelación y ordenación de cálculos modales así como de su posible jerarquización- está aún hoy en día abierto, no podemos negar la relevancia que el trilema de Diodoro podría llegar a jugar en un contexto contemporáneo de discusión de filosofía de la lógica modal.

\section{Hacia una aproximación modal alética de la lógica megárico-estoica}

He mencionado en numerosas ocasiones la estrecha relación entre la relación de accesibilidad o los diferentes cálculos modales con las propuestas y discusiones megáricoestoicas. Desarrollemos un poco más el fundamento semántico de un cálculo modal normal contemporáneo antes de proponer una formalización más adecuada de los operadores modales de necesidad y posibilidad en el sentido estoico.

Una semántica modal -sin cuantificar, para no complicarnos excesivamente- no es más que un cálculo proposicional normal en el que, además de una función veritativa $v$, se añaden un conjunto de mundos o estados posibles $W$ y una relación de accesibilidad, $R$, capaz de interrelacionar estos. También, debo destacar que existe siempre un mundo real o existente actual-, desde el cual hablamos, a partir del cual se determinará cuáles le son accesibles -cuyo contenido es afirmable y modalmente relevantey cuáles no. Por tanto, se construye una tripla 
$<W, R, v>$ para poder realizar cualquier tipo de evaluación.

La proposición primera del trilema, «ingenua» en un sentido modal para Benítez (2015 p. 134), supone que el pasado es inalterable. Una crítica a esta postura desde una perspectiva actual sería la de no tener en cuenta que existen, desde el mundo actual, pasados posibles o contingentemente concebibles que queden automáticamente descartados, pero no es baladí el posicionamiento de Diodoro al rechazar 1).

Lo que implica esto no es que se elimine la posibilidad de pasados contingentes, sino que se suprima su posibilidad en cuanto que tal. Se asume así una causalidad -muy similar a la que encontramos en lo que en mecánica relativista acostumbra a llamarse línea de vida de una partícula- de forma que toda contingencia del pasado se da en cuanto que el marco de accesibilidad es dinámico y en algún momento los estados pasados fueron, precisamente, actuales o presentes.

Sin asumir ningún compromiso con la metáfora del flujo del tiempo en los términos en que Prior la asume, se puede concebir un marco de discrecionalidad metafísica que asume que si un suceso ha tenido lugar desde el mundo actual este suceso es necesario por la vinculación causal con este mismo mundo. Sin embargo, si se tomara como mundo actual aquel temporalmente anterior, sí que se admitiría su posibilidad. Y frente a la réplica que pasase por asumir que dicho futuro, entonces, sería inaccesible para el estado actual, cabría invocar la capacidad predictiva. La enunciación de un contenido proposicional aseverable en un estado posible en cuanto que futuro puede ser lo suficientemente vaga para determinarse en cuanto que tal.

No obstante, la verdadera clave reside, en último término, en observar que los estados en cuanto que momentos o instantes espaciotemporales, solamente constituyen un pequeño caso, una restricción teórica sin duda útil -es por ejemplo, el caso de los observables en mecánica relativista operando como estados posibles accesibles o no entre sí cuando este caso se restringe, en concreto, a la interacción entre dos marcos, piénsese en un diagrama de Minkowski, causalmente conectados por un cono de luz-, pero que no puede ni debe ser tomada como la generalización metafísica que determine, en último término, la caracterización final de los operadores de necesidad y posibilidad en una semántica modal.

\section{Conclusiones}

Por todo lo anterior, no puedo sino concluir que parece, cuanto menos, útil realizar una aproximación a los desarrollos modales megárico-estoicos a la luz de los resultados obtenidos en contextos técnicos de estudio de cálculos modales desde semánticas relacionales. Utilidad, por otro lado, doble.

En primer lugar, se podría así superar el análisis sesgado e inconsistente, mayoritario hasta la fecha, basado en la inclusión de variables temporales ad hoc llegando a obtener una mejor comprensión de las discusiones metafísicas operantes de fondo en las discusiones megáricoestoicas -es el caso, entre otros, del esclarecimiento metafísico del trilema de Diodoro. Tomar las definiciones de necesidad y posibilidad, así como sus negaciones en cuanto que modalidades aléticas, permite esclarecer el fondo metafísico de las semánticas megáricoestoicas reabriendo una gran cantidad de debates hermenéuticos en el seno de la historia de la filosofía antigua.

En segundo lugar, dentro de dicho ejercicio de clarificación conceptual, está claro que las discusiones derivadas de dichas semánticas revisten una enorme relevancia y actualidad en el seno de la llamada metafísica modal.

Finalmente, existen una serie de trabajos, entre los que destaca Goldbatt (1980), que han pasado por aplicar las definiciones de Diodoro en contextos de discusión interdisciplinar relevantes hoy en día. Sin duda alguna, la relectura de dichas definiciones desde una semántica relacional como modalidades aléticas -abandonando su forzada inclusión en lógicas temporales- alentará una serie de investigaciones aplicativas muy prometedoras. 


\section{Referencias}

Benítez, A. (2015). Lógicas no clásicas. Una introducción. Escolar y Mayo Editores.

Bocheński, I. M. (1963). Ancient Formal Logic. North-Holland Publish Co.

Coxon, A. H. (1986). The fragments of Parmenides. A critical text with introduction, the ancient testimonia and commentary. Van Gorcum.

de Harven, V. (2016). Necessity, Possibility and Determinism in Stoic Thought en Cresswell, M., Mares, E. y Rini, A., Logical Modalities from Aristotle to Carnap: The Story of Necessity (pp. 70-90). Cambridge University Press.

Diels, H. (1906). Die Fragmente der Vorsokratiker. Weidmannsche Buchhandlung y Kranz, W. ed. (1952), sexta edición revisada con adiciones e índice.

Goldblatt, R. (1980). Diodorean modality in Minkowski spacetime. Studia Logica, 39, pp. 219236.

Hintikka, J. (1962). Knowledge and Belief. Cornell University Press.

KnealeW. y M. (1962). The Development of Logic. Oxford University Press.

Kripke, S. (1963). Semantical Considerations on Modal Logic. Acta Philosophica Fennica, 16, pp. 83-94.

Lewis, C. I. (1918). A Survey of Symbolic Logic. University of California Press.

Mates, B. (1973). Lógica de los Estoicos. (M. García Baró, Trad.). Tecnos. (Obra original publicado en 1985)

Prior, A. (1955). Diodoran Modalities. Philosophical Quarterly, vol. 5, pp. 205-213.

Las fuentes clásicas no especificadas se citan en su edición canónica:

Damascio, De principiis.

Simplicio, Física.

Teofrasto, Physikoi dóxai.

Alejandro de Afrodisias, Comentario a los Analíticos Primeros de Aristóteles Libro Primero, I.

Aristóteles, Sobre la interpretación.

-Analíticos primeros.

-Metafísica. 